\title{
Outcomes and Prognostic Factors of Neuroendocrine Cervical Cancer in the National Cancer Institute of Thailand: A Retrospective Cohort Study
}

\author{
Suwaree Siripaopradit ${ }^{1}$, Supakorn Pitakkarnkul ${ }^{1}$, Panwad Ratanasrithong ${ }^{1}$, \\ Sujira Foongfaung ${ }^{2}$
}

${ }^{1}$ Department of Gynecologic Oncology, National Cancer Institute, Bangkok, Thailand. ${ }^{2}$ Borommarajonani College of Nursing, Changwat Nonthaburi, Praboromrajchanok Institute, Thailand.

\begin{abstract}
Background: Neuroendocrine carcinoma of the cervix (NECC) is a rare tumor with an aggressive natural course. Objective: This study was to investigate the outcomes and prognostic factors for NECC patients who had been treated at the National Cancer Institute of Thailand (NCI). Methods: NECC patients who had been treated between January 2008 to December 2017 at NCI of Thailand were review. Data were collected from medical records, tumor registry files, and civil registration system. The impacts of clinical and pathologic risk factors on the overall survival (OS) and progression-free survival (PFS) were evaluated using Kaplan-Meier survival analyses and the prognostic factor was determined by univariate Cox regression analysis. Result: Thirty-three NECC patients including 12 patients early-stage patients (FIGO stage I-IIA) and 21 patients advanced stage patients (FIGO stage IIB-IVA) were reviewed. All of the patients in the early stage underwent radical hysterectomy and lymph node dissection (RHND) with or without neoadjuvant chemotherapy. Ten patients received adjuvant therapy with pelvic radiation and/or cisplatin-etoposide following operation. Median OS and PFS among early-stage patients were 25.69 months and 18.65 months, respectively. Treatments among patients with advanced-stage included initial chemotherapy, initial concurrent chemoradiation (CCRT), and only CCRT. Patients receiving initial chemotherapy had better PFS (81.4 months) and OS (81.4 months) compared to those who received initial CCRT (PFS 9.0 months; OS 13.2 months; $\mathrm{P}=0.013$ ) or only CCRT (PFS 13.0 months; OS 16.1 months; $\mathrm{P}=0.016$ ). No statistically significant factors associated with survival were noted. Conclusion: Patients with NECC carried a poor prognosis even in the early stage of the disease. Systemic chemotherapy may be required for all stages of this highly aggressive cancer.
\end{abstract}

Keywords: Neuroendocrine carcinoma of the cervix- treatment- prognosis

Asian Pac J Cancer Care, 5 (1), 51-56

Submission Date: 11/21/2019 Acceptance Date: 01/17/2020

\section{Introduction}

Cervical cancer is the third most common female cancer in Thailand. There are 11.7 new cervical cancer cases per 100,000 women population per year [1]. Neuroendocrine carcinoma of the cervix (NECC) is a rare histological type of cervical cancer, accounting for approximately $1.5 \%$ of all cervical cancers [2,3-4]. The diagnosis of NECC can be made on a detailed histopathologic examination. Positive immunohistochemical staining for neuroendocrine markers like synaptophysin (SYN), chromogranin (CHG),

CD56 (N-CAM), and neuron-specific enolase (NSE) is useful to support the diagnosis of NECC was subdivided by World Health Organization (WHO) Classification in low-grade neuroendocrine tumors (NET) include typical carcinoid and atypical carcinoid tumors and high-grade neuroendocrine carcinoma (NEC) include small cell NEC and large cell NEC [3-4].

NECC derived from neuroendocrine cells that originate from the embryonic neuroectoderm and display

Corresponding Author:

Dr. Suwaree Siripaopradit

Department of Gynecologic Oncology, National Cancer Institute, Bangkok, Thailand.

Email: sirisuwaree@gmail.com 
an immunohistochemical profile consistent with endocrine glandular cells [2]. NECC is HPV- related cancer. Castle et al (2018) found that $88 \%$ of NECC were HPV positive. The predominant subtypes were HPV type 16 and 18 . HPV infection, therefore, is the underlying cause for most cases of NECC and that could be prevented by prophylactic HPV vaccination [5].

NECC is likely to invade the lymph-vascular space and to spread to the regional lymph node at the time of diagnosis. Also, relapses occur more often in NECC leading to poor prognosis when compared to squamous cell carcinoma and adenocarcinoma of the cervix [2-3]. Due to the rarity of this malignancy, appropriate management of NECC remains inconclusive. At the present, management of NECC is guided by the treatment outcomes of neuroendocrine tumors in other organs i.e. pancreas and lung [6].

To date, there is a lack of prospective and randomized trials to establish management guidelines for NECC. In 2011, the Society of Gynecologic Oncology (SGO) issued a clinical document summarizing the available literature on NET of the female reproductive tract [7]. In 2014, the Gynecologic Cancer InterGroup (GCIG) published a consensus review for NECC treatment [8]. These guidelines recommend using multimodality therapeutic strategies including surgery, radiotherapy, and systemic chemotherapy for this aggressive tumor. Various chemotherapy regimens have been advocated by SGO and GCIG schemes including cisplatin/etoposide, cisplatin/ irinotecan, cisplatin/vincristine/bleomycin.

This study was undertaken to determine the clinical characteristics and treatment outcomes of patients with NECC who had been diagnosed and treated at NCI of Thailand. An effort to discern any pattern or characteristics of patients with a higher risk of poor treatment outcomes may provide foundational data for designing effective treatment for NECC.

\section{Materials and Methods}

\section{Patients selection}

This study was a retrospective cohort study. There were 42 patients who had been diagnosed with NECC at NCI of Thailand between January 1, 2008 to December 31,2017 . We included only patients who were able to complete initial treatment and had pathological slides available for reviewing by NCI pathologists in order to confirm the diagnosis. Nine patients were excluded from study. Three patients had incomplete data, two patients had incomplete initial treatment, two patients were not received treatment at the study institution and two patients had other pathologic diagnosis after pathologic reviewing, leaving 33 patients for further analyses. Data were abstracted from medical records, tumor registry files, and Thailand civil registration system.

\section{Patients, clinicopathological and treatment variables}

All pathological slides were reviewed by NCI pathologist. Additional immunohistochemical stains were performed in almost all of the cases (27 patients, $81.2 \%$ ) to confirm the diagnosis of NECC.

All patients underwent a staging procedure which includes a physical examination, blood for BUN, creatinine, liver function test, chest roentgenogram, and computerized tomography of abdominopelvic area. Cystoscopy and proctoscopy were performed in some selected cases. Tumors were staged according to the 2018 International Federation of Gynecology and Obstetrics Clinical Staging Criteria for cervical carcinoma. Status of pelvic and para-aortic lymph node was assessed based on pathological diagnosis in operated patients or based on computerized tomography scan finding in non-operable patients. The early and advanced stages were defined as stages I-IIA and stages IIB-IVA, respectively.

\section{Outcomes measurement}

The primary endpoints were progression-free survival (PFS) and overall survival (OS). Secondary endpoints were clinicopathological findings and factors associated with the response of treatment. The sites of the initial recurrence were classified as locoregional and distant recurrences. PFS was calculated from the date of start initial treatment to recurrence or death. OS was calculated from the start of initial treatment to death.

SPSS version 22 was used. The survival was evaluated using the Kaplan-Meier survival analysis and the survival differences were compared using a log-rank test. The potential prognostic factor associated with treatment outcome was determined by univariate Cox regression analysis. $\mathrm{P}<0.05$ were considered as statistically significant.

\section{Results}

Study participants

A total of 33 NECC patients were reviewed. The median follows up time of the entire cohort was 27.37 months (2.63-111). The median patients' age was 47 years (range 25-73 years). All patients presented with abnormal vaginal bleeding. The clinicopathological and treatment characteristics of the patients are shown in Table 1.

Twelve patients had been diagnosed with early-stage cancer while the remaining 21 patients had advanced-stage disease. Most of the patients (24 patients, $72.7 \%$ ) had a tumor size of $4 \mathrm{~cm}$ or larger. Twenty-nine $(87.9 \%)$ patients had been diagnosed with small cell carcinoma histology. Carcinoid tumors were not found in this study. Ten patients had other histological types of cancer mixed with NECC including 7 adenocarcinoma and 3 squamous cell carcinoma.

\section{Treatment outcomes in the early stages}

The stages of 12 patients included 6 IB2, 5 IB3, and 1 IIA. All patients with early-stage had undergone radical hysterectomy with pelvic node dissection (RHND) with or without neoadjuvant chemotherapy as their initial treatments. Neoadjuvant chemotherapy (NAC) regimens used in the study institution were single cisplatin (60-80 mg/m² every 3 weeks, 2 cycles) and combination of cisplatin and etoposide (cisplatin with $60-80 \mathrm{mg} / \mathrm{m}^{2}$ given 
Table 1. Patients Clinicopathologic Characteristics $(\mathrm{N}=33)$

\begin{tabular}{|c|c|c|}
\hline Variables & $\mathrm{n}$ & $\%$ \\
\hline \multicolumn{3}{|l|}{ Stage* } \\
\hline IB-IIA & 12 & 36.4 \\
\hline IIB-IVA & 21 & 63.6 \\
\hline \multicolumn{3}{|l|}{ Tumor size (cms) } \\
\hline$<4$ & 9 & 27.3 \\
\hline$\geq 4$ & 24 & 72.7 \\
\hline \multicolumn{3}{|l|}{ Histologic type of NEC } \\
\hline Small cell carcinoma & 29 & 87.9 \\
\hline Large cell carcinoma & 4 & 12.1 \\
\hline \multicolumn{3}{|l|}{ Histologic homology } \\
\hline Pure & 23 & 69.7 \\
\hline Mixed & 10 & 30.3 \\
\hline \multicolumn{3}{|l|}{ Lymph node involvement } \\
\hline No LN involvement & 16 & 48.48 \\
\hline Pelvic LN alone & 4 & 12.12 \\
\hline Para-aortic LN alone & 2 & 6.06 \\
\hline Both & 11 & 33.33 \\
\hline \multicolumn{3}{|l|}{ Immunohistochemistry } \\
\hline Yes & 27 & 81.8 \\
\hline No & 6 & 18.2 \\
\hline \multicolumn{3}{|c|}{ Initial treatment modalities Early-stage: stage IB- IIA $(n=12)$} \\
\hline $\mathrm{NAC} \longrightarrow \mathrm{RHND} \pm \mathrm{CMT} / \mathrm{CCRT}$ & 5 & 41.7 \\
\hline RHND initial $(n=7)$ & 7 & 58.3 \\
\hline \multicolumn{3}{|l|}{ Advanced-stage: stage IIB-IVA ( $\mathrm{n}=21$ ) } \\
\hline $\mathrm{CMT} \longrightarrow \mathrm{RT} / \mathrm{CCRT}$ & 7 & 33.3 \\
\hline $\mathrm{CCRT} \longrightarrow \mathrm{CMT}$ & 7 & 33.3 \\
\hline CCRT & 7 & 33.3 \\
\hline
\end{tabular}

*Stage by the FIGO2018(International Federation of Gynecology and Obstetrics staging system) [9]; NAC, Neoadjuvant Chemotherapy; RHND, Radical hysterectomy with pelvic node dissection; CMT, Chemotherapy; RT, Radiotherapy; CCRT, Concurrent chemoradiation

on the first day of every 3 weeks cycle and etoposide with $100 \mathrm{mg} / \mathrm{m}^{2}$ given on the first three days of every 3 weeks, 2 cycles) is shown in Table 1.

Table 2 displays the associations between baseline clinicopathologic factors and survival by mean of univariate analyses. No significant factors associated with either PFS and OS were noted in this cohort.

The median OS and PFS of early-stage patients were 25.69 months and 18.65 months, respectively. There was no survival difference between patients receiving NAC and those who did not $(\mathrm{P}=0.528$ for $\mathrm{OS}$ and $\mathrm{P}=0.554$ for PFS) as shown in Table 2.

For the patients who received RHND, three of seven patients who received adjuvant chemotherapy alive at the end of study time. These patients were noted to have a tumor size of $4 \mathrm{~cm}$ or smaller. Furthermore, one of these three women had ovarian metastasis which had been treated by cytoreductive surgery and adjuvant combination chemotherapy and was still alive without disease at the time of the last follow-up.
Two patients who underwent postoperative CCRT and received four cycles of cisplatin-etoposide. They had more than $4 \mathrm{~cm}$ of tumor size and pelvic lymph node involvement. Both of them died from disease at 18.50 months and 15.50 months later.

Two patients who did not receive any postoperative adjuvant treatment, one died after disease recurrence. The remaining one patient with a tumor size of less than $4 \mathrm{~cm}$ who received two cycles of cisplatin/etoposide for NAC was still alive without recurrence.

One patient who underwent only postoperative CCRT died from disease at 13.13 months after treatment.

\section{Treatment outcomes in advance stages}

Stages of cancer among this group included 5 IIB, 4 IIIB, 2 IIIC1, 9 IIIC2r, and 1 IIIC2p. Lymph node metastasis was diagnosed when the axial plain diameter of the suspected lymph node on CT was $1 \mathrm{~cm}$ or larger [10]. Lymph node status was not a significant factor affecting survival among patients with advanced-stage NECC (Table 2; $\mathrm{P}=0.157$ for PFS; $\mathrm{P}=0.249$ for $\mathrm{OS}$ ).

In this study, patients with advanced-stage were divided into 3 groups by types of initial treatment including, first: patients receiving initial chemotherapy followed by radiation alone or CCRT (seven patients included 2 stage IIIB, 4 IIIC, and 1 IVA), second: patients receiving CCRT followed by 3-4 cycles of cisplatin/etoposide combination chemotherapy (seven patients included 3 stage IIB, 4 IIIC) and third: patients receiving CCRT alone (seven patients included 1stage IIB,2 IIIB, and 4 IIIC).

Patients receiving initial chemotherapy had the longest median PFS and OS (81.37 months for both outcomes) compared to those who received other treatments ( $\mathrm{P}=0.013$ for median PFS and $\mathrm{P}=0.016$ for $\mathrm{OS})$ is shown in Table 2. All patients with stage IIIC or IVA were able to complete their treatment with four cycles of cisplatin/ etoposide, followed by pelvic radiotherapy. Five of seven patients who receiving initial chemotherapy were no evidence of recurrence at the last follow-up. The remaining two patients experienced pelvic and periaortic lymph node metastases died of disease.

In initial CCRT followed by chemotherapy group, one of two patients with stage IIB who were alive had ovarian metastasis. The remaining patient had no evidence of recurrent disease. Five patients who died from the disease were in stage IIIB or IIIC. Two patients who were still alive after receiving CCRT alone were in stage IIB. One of these two patients had adenocarcinoma with focal NECC differentiation on histological examination.

Median PFS and OS of the entire cohort were 20.27 months (95\% CI 0.00-46.94) and 81.37 months (95\% CI 45.98-117.24), respectively. Median PFS and OS were 18.65 months and 25.69 months for early-stage and 25.69 months and 28.93 months for the advanced stage, respectively (Figure 1). The stage of disease was not significantly associated with survival $(\mathrm{P}=0.343$ for $\mathrm{PFS}$ and $\mathrm{P}=0.291$ for $\mathrm{OS})$.

Twenty patients $(64.51 \%)$ were noted to experience disease recurrence. The median time to recurrence was 20.27 months $(95 \%$ CI 0.00 - 46.94). The sites of 
Table 2. Univariate Statistics by Progression Free Survival and Overall Survival $(\mathrm{N}=33)$

\begin{tabular}{|c|c|c|c|c|}
\hline Variables & Median PFS(mo) & p-value & Median OS(mo) & p-value \\
\hline \multicolumn{5}{|l|}{ Stage } \\
\hline Early stage: IB2-IIA & 18.65 & & 25.69 & \\
\hline Advance stage: IIB-IVA & 28.93 & 0.343 & 28.93 & 0.291 \\
\hline \multicolumn{5}{|l|}{ Tumor size $(\mathrm{cm})$} \\
\hline$<4$ & 20.27 & & 31.93 & \\
\hline$\geq 4$ & 18.27 & 0.173 & 22.25 & 0.349 \\
\hline \multicolumn{5}{|l|}{ Histology of NEC } \\
\hline Small cell carcinoma & 20.27 & & 31.93 & \\
\hline Large cell carcinoma & 18.27 & 0.280 & 14.63 & 0.162 \\
\hline \multicolumn{5}{|l|}{ Histologic homology } \\
\hline Pure & 20.27 & & 28.93 & \\
\hline Mixed & 18.27 & 0.540 & 25.10 & 0.528 \\
\hline \multicolumn{5}{|l|}{ Lymph node involvement } \\
\hline No & 23.28 & & 30.43 & \\
\hline Pelvic or Para-aortic metastases & 13.00 & 0.157 & 18.80 & 0.249 \\
\hline \multicolumn{5}{|l|}{ Treatment modalities } \\
\hline \multicolumn{5}{|l|}{ Early stage: stage IIB-IVA $(\mathrm{n}=12)$} \\
\hline NAC $\rightarrow$ RHND initial $(n=5)$ & 18.80 & & 24.00 & \\
\hline RHND initial (n=7) & 18.50 & 0.554 & 27.37 & 0.604 \\
\hline \multicolumn{5}{|l|}{ Advance stage: stage IIB-IVA $(n=21)$} \\
\hline $\mathrm{CMT} \longrightarrow \mathrm{CCRT}(\mathrm{n}=7)$ & 81.37 & & 81.37 & \\
\hline $\mathrm{CCRT} \longrightarrow \mathrm{CMT}(\mathrm{n}=7)$ & 9.00 & & 13.17 & \\
\hline $\operatorname{CCRT}(\mathrm{n}=7)$ & 13.00 & 0.013 & 16.13 & 0.016 \\
\hline
\end{tabular}

recurrence are summarized shown in Table 3.

Almost of recurrences were distant metastasis (18 in $20,90.0 \%)$. Lung was the most common site of metastasis (16 in 20,80.0\%), followed by liver (6 in 20, 30.0\%). The other distant metastatic sites included 1 pancreas, 1 skin, 1 supraclavicular lymph node. Four patients had loco-regional metastatic sites included 2 ovary and 2 vagina. All of the patients with 2 vaginal recurrence did not receive postoperative pelvic radiation. Eleven

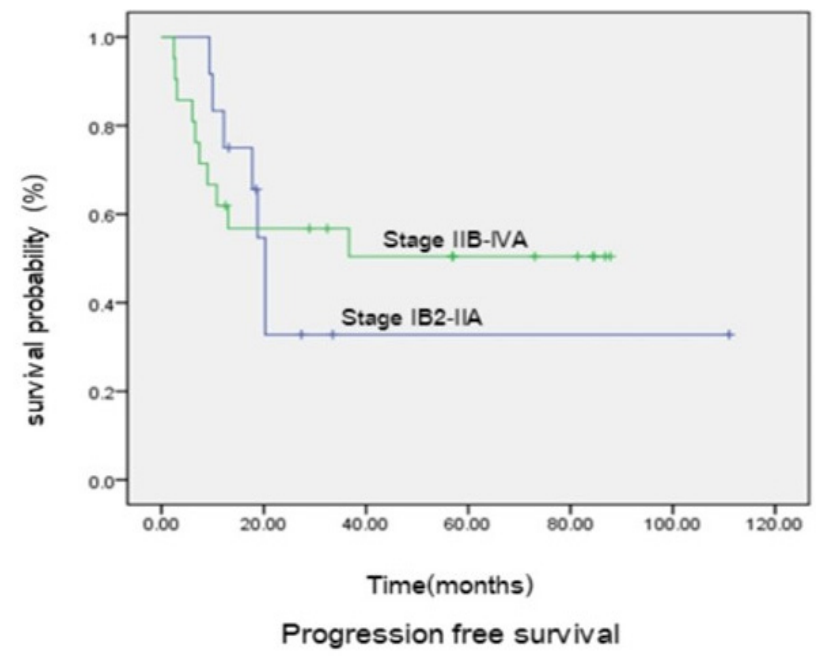

$(55.0 \%)$ patients had multiple sites of metastasis is shown in Table 3.

\section{Discussion}

Our data represent the grave prognosis of NECC. The median PFS and OS of the entire cohort were only 20.27 months and 81.37 months thus supporting that $\mathrm{NECC}$ is an aggressive cervical cancer. In a pooled

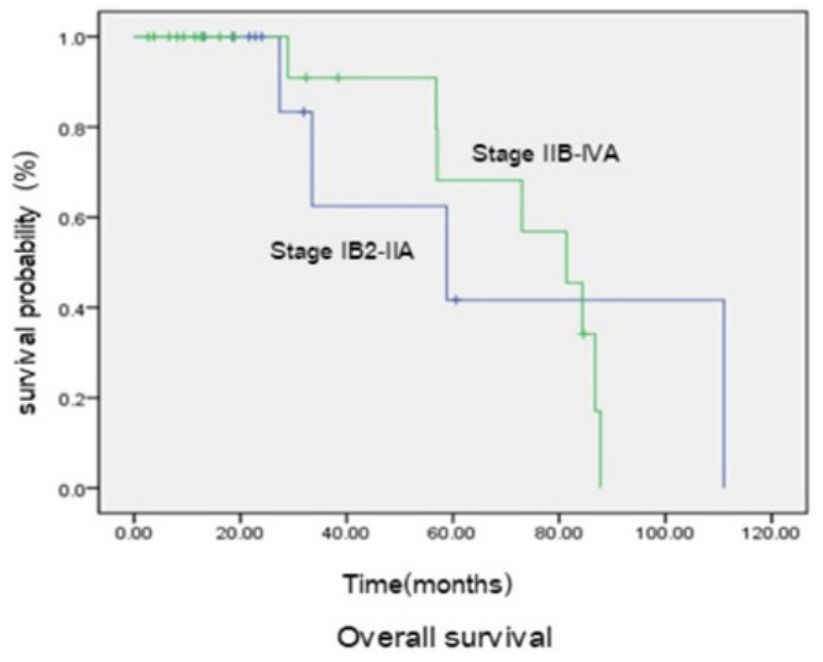

Figure 1. Progression-free Survival and Overall Survival on the International Federation of Gynecology and Obstetrics (FIGO) Stage 
Table 3. Metastatic Site by Treatment in early and Advanced Stage $(\mathrm{N}=31)$

\begin{tabular}{|c|c|c|c|c|c|c|}
\hline & \multicolumn{2}{|c|}{ Early stage } & \multicolumn{2}{|c|}{ Advance stage } & \multirow[t]{2}{*}{ Total } & \multirow[t]{2}{*}{$\%$} \\
\hline & + Adj & No Adj & iCMT & iCCRT & & \\
\hline \multicolumn{7}{|c|}{ Distant metastasis } \\
\hline Lung & 6 & 1 & 0 & 9 & 16 & 80.0 \\
\hline Liver & 2 & 0 & 0 & 4 & 6 & 30.0 \\
\hline Brain & 0 & 0 & 0 & 2 & 2 & 10.0 \\
\hline Skin/SCLN & 0 & 0 & 0 & 2 & 2 & 10.0 \\
\hline Pancreas & 0 & 0 & 0 & 1 & 1 & 5.0 \\
\hline \multicolumn{7}{|c|}{ Loco-regional metastasis } \\
\hline Ovary & 1 & 1 & 0 & 0 & 2 & 10.0 \\
\hline Vagina & 1 & 1 & 0 & 0 & 2 & 10.0 \\
\hline
\end{tabular}

No data of metastases 2 patients in advanced stage who received initial treatment with chemotherapy. The number of metastatic patients $20=64.52 \%$ Multiple metastatic site $11=55.0 \%$; Non metastatic patients. $11=35.48 \%$; Abbreviations, +Adj, with postoperative adjuvant treatment; No Adj, without postoperative adjuvant treatment; iCMT, initial treatment with chemotherapy; iCCRT, initial treatment with concurrent chemoradiation.

analysis of published studies, the 2-year and 5-year OS of NECC patients were only $50 \%$ and $34 \%$, respectively [11].

In the literature, advanced stage, tumor size, number of lymph node metastases, pure small cell histology, and smoking have been linked to a worse clinical outcome for NECC [12-13]. In this study, there were no associations between these clinicopathological factors and survival. The small number of patients available for this study may preclude our ability to note the impact of these factors on survival.

A systematic review assessing 3,538 cases of NECC in 112 included studies found that no retrospective or prospective comparison of the efficacy of surgery-based, chemotherapy-based and radiotherapy-based treatment schemes within comparable disease stages in the published studies. Although there is no standard chemotherapy regimen for NECC, cisplatin or carboplatin plus etoposide (EP) was the most commonly used regimen [11].

For early-stage NECC, all patients in our study underwent RHND with or without NAC. There was no survival difference between patients receiving NAC and those who did not $(\mathrm{P}=0.554$ for $\mathrm{PFS}$ and $\mathrm{P}=0.604$ for OS; Table 3). Patients who received postoperative chemotherapy appeared to have better survival when compared to those who received other adjuvant treatments (Figure 1) which might suggest that adjuvant chemotherapy following RHND for early-stage NECC may be more preferred. Lee et al. (2008) reported no benefit in overall survival among six patients who received NAC [14]. Wang et al. (2012) assessing 179 NECC patients found that for stage IA-IIB primary surgical treatment for stage IA-IIB had a trend of worse PFS $(41.2 \%$ versus $60.5 \%, \mathrm{p}$ $=0.086)$ compared to other treatments [15]. On the other hand, Ishikawa et al. (2018) reviewing 93 stages I-II NECC patients found that patients who underwent radical surgery had better overall survival than those who received definitive radiotherapy $(\mathrm{P}=0.043)$ [16]. In addition, the previous study noted that radical surgery followed by adjuvant chemotherapy decreased the risk of recurrence and showed a trend toward improved survival [4]. These previous findings together with our results may support the role of adjuvant chemotherapy following radical surgery among patients with early-stage NECC as having been recommended in SGO and GCIG guidelines [7-8].

For locally advanced disease, our study represents better survival outcomes among patients receiving chemotherapy as their initial treatment compared to other treatment modality $(\mathrm{PFS}=81.37, \mathrm{P}=0.013$ and $\mathrm{OS}=81.37, \mathrm{P}=0.016$ ). Wang et al. (2012) also noted that primary treatment containing cisplatin-etoposide for advanced stage NECC was associated with significant better 5-year PFS (42.9\% versus $11.8 \%, \mathrm{P}=0.041)$ and OS ( $45.6 \%$ versus $17.1 \%, \mathrm{P}=0.035)$. Starting treatment with systemic chemotherapy in a timely fashion may effectively eradicate the occult metastatic lesion which could have resulted in better tumor control than other treatments [15].

An unexpected result in our study which was contrast to previously reported findings were the comparable survival outcomes among patients with early and advanced stages NECC [11, 12-17]. Our findings may suggest that the routine procedure used for staging cervical cancer may be inappropriate for NECC. NECC is aggressive cancer and highly likely to spread outside the cervix even in patients with clinically early stages of the disease. In our study, the staging procedure was applied as per the recommendation of FIGO staging criteria [9]. However, SGO suggests using positron emission tomography (PET)/ CT scan other than CT scan for assessing patients with NECC [7]. At MD Anderson Cancer Center, PET/CT scan is considered as initial radiological staging for this aggressive tumor [18]. Inappropriate staging of NECC can lead to inadequate assessment of patients with clinically early stage and thus may lead to receiving insufficient treatment.

In our study, three patients who developed vaginal stump recurrence did not receive postoperative pelvic radiation. However, the role of pelvic radiation among NECC may be limited as all of the patients with recurrence were noted to have distant metastasis.

A small number of NECC patients which is secondary to an extreme rarity of this cancer is a major limitation of this study and precluded our ability to draw any meaningful conclusion. However, this study suggested the role of 
chemotherapy for all stages NECC. The strength of this study is that the pathological slides were reviewed and the majority of patients had immunostaining to confirm the diagnosis of NECC.

In conclusion, NECC may need more meticulous metastatic workup than usual type cervical cancer. Chemotherapy may be required for all patients with NECC even among those who have clinically early stages.

\section{Acknowledgments}

Authors appreciate all supports from the National Cancer Institute of Thailand (NCI), patients and all the staff of all clinics involving in this study.

\section{References}

1. Imsamran W, Pattatang A, Supattagorn P, Chiawiriyabunya I, Namthaisong K, Wongsena $\mathrm{M}$ et al. Cancer in Thailand Vol. IX, 2013-2015, New Thammada Press, Bangkok:Thailand, 2018:47-50.

2. Gadducci A, Carinelli S, Aletti G.(2017). Neuroendocrine tumors of the uterine cervix: a therapeutic challenge for gynecologic oncologists. Gynecol Oncol. 2017;144:637-46.

3. Kim JY, Hong SM, Ro JY. Recent updates on the grading and classification of neuroendocrine tumors. Ann Diag Pathol. 2017;29:11-6.

4. Kurman RJ, Carcangiu ML, Herrington CS, Young RH. Tumors of the uterine cervix. WHO classification of tumors of the female reproductive organs. Lyon IARC Press, 2014:169-206.

5. Castle PE, Pierz A, Stoler MH. A systematic review and meta-analysis on the attribution of human papillomavirus (HPV) in neuroendocrine cancers of the cervix. Gynecol Oncol. 2018;148:422-9.

6. Grande E, Capdevila J, Castellano D, Teulé A, Durán I, Fuster $\mathrm{J}$ et al. Pazopanib in pretreated advanced neuroendocrine tumors: a phase II, open-label trial of the Spanish task force Group for Neuroendocrine Tumors (GETNE). Ann Oncol. 2015;26:1987-93.

7. Gardner GJ, Reidy-Lagunes D, Gehrig PA. Neuroendocrine tumors of the gynecologic tract: a Society of Gynecologic Oncology (SGO) clinical document. Gynecol Oncol, 2011;122:190-8.

8. Satoh T, Takei Y, Treilleux I, Devouassoux-Shisheboran M, Ledermann J, Viswanathan AN et al. Gynecologic Cancer InterGroup (GCIG) consensus review for small cell carcinoma of the cervix. Int J Gynecol Cancer. 2014;24:S102-8.

9. Bhatla N, Aoki D, Sharma DN, Sankaranarayanan R. Cancer of the cervix uteri. Int J Gynecol Obstet. 2018;143(Suppl.3):22-36.

10. Eisenhauer EA, Therasse P, Bogaerts J, Schwartz LH, Sargent D et al. New response evaluation criteria in solid tumors: revised RECIST guideline(version1 .1). Eur J Cancer. 2009;45:228-47.

11. Tempfer CB, Tischoff I, Dogan A, Hilal Z, Schultheis B, Kern $P$ et al. Neuroendocrine carcinoma of the cervix: a systematic review of the literature. BMC Cancer. 2018;18:1-16.

12. Chan JK, Loizzi V, Burger RA, Rutgers J, Monk BJ. Prognostic factors in neuroendocrine small cell cervical carcinoma: a multivariate analysis. Cancer. 2003;97:568-74.

13. Viswanathan AN, Deavers MT, Jhingran A, Ramirez PT, Levenback C, Eifel PJ. Small cell neuroendocrine carcinoma of the cervix: outcome and patterns of recurrence. Gynecol
Oncol. 2004;93:27-33.

14. Lee JM, Lee KB, Nam JH, Ryu SY, Bae DS, Park JT et al. Prognostic factors in FIGO stage IB-IIA small cell neuroendocrine carcinoma of the uterine cervix treated surgically: results of a multi-center retrospective Korean study. Ann Oncol. 2008;19:321-6.

15. Wang KL, Chang TC, Jung SM, Chen $\mathrm{CH}$, Cheng YM, $\mathrm{Wu} \mathrm{HH}$ et al. Primary treatment and prognostic factors of small cell neuroendocrine carcinoma of the uterine cervix: a Taiwanese Gynecologic Oncology Group study. Eur J Cancer. 2012;48:1484-94.

16. Ishikawa M, Kasamatsu T, Tsuda H, Fukunaga M, Sakamoto A, Kaku T et al. Prognostic factors and optimal therapy for stages I-II neuroendocrine carcinomas of the uterine cervix: a multi-center retrospective study. Gynecol Oncol. 2018;148:139-46.

17. Roy S, Ko JJ, Bahl, G. Small cell carcinoma of cervix: A population-based study evaluating $\mathrm{T}$ standardized provincial treatment protocols. Gynecol Oncol Rep. 2019; 27:54-9.

18. Salvo G, Gonzalez MA, Gonzales NR, Frumovitz M. Updates and management algorithm for neuroendocrine tumors of the uterine cervix. Int J Gynecol Cancer. 2019;29:986-95.

This work is licensed under a Creative Commons AttributionNon Commercial 4.0 International License. 